\title{
Remote monitoring of nuclear power plants in Baden-Wuerttemberg - From measurement to emergency protection
}

\author{
T. WILBOIS ${ }^{1}$, Y. REN ${ }^{1}$, U. NEFF ${ }^{2}$, W. SCHEUERMANN ${ }^{3}$, C. GRIMM ${ }^{4}$, \\ M. HAGMANN ${ }^{4}$
}

\begin{abstract}
The nuclear reactor remote monitoring system of the federal state of BadenWuerttemberg (KFUe BW) is realized according to the renewed "recommendations for remote monitoring of nuclear power plants" (BMU, 2005a). The spectrum of the system covers both, pursuit of operational procedures, and incidents or accidents. The KFUe BW provides a measurement network and information system for operational parameters at the plant sites as well as radiological and meteorological measurements in their vicinity. For the Ministry, it preferentially serves as an instrument of nuclear supervision. This paper gives a brief survey of the system architecture and concentrates on the role of the KFUe with respect to the determination and evaluation of the radiological situation in the range of off-site emergency management.
\end{abstract}

Keywords: Environmental monitoring/nuclear accident/radiological emergency

\section{Introduction}

As part of its responsibilities as nuclear supervisory authority, the Ministry of the Environment, Climate Protection and the Energy Sector Baden-Württemberg (UM) operates a computer-based system for remote monitoring of nuclear power plants (KFUe BW). In addition to the Baden-Wuerttemberg nuclear power plants Philippsburg, Neckarwestheim and Obrigheim, also the foreign locations close to the border area, i.e. Fessenheim in France and Leibstadt and Beznau in Switzerland, are monitored. The system is realized according to the renewed "recommendations for remote monitoring of nuclear power plants" (BMU, 2005a). This

\footnotetext{
T-Systems GEI GmbH, Systems Integration, 89070 Ulm, Germany.

${ }^{2}$ LUBW Regional Office for Environment, Measurements, and Nature Conservation Baden-Wuerttemberg, Department 32 radioactivity, radiation protection, Griesbach Straße 1, 76185 Karlsruhe, Germany.

University of Stuttgart, Institute of nuclear technology, Pfaffenwaldring 31, Germany.

${ }^{4}$ Ministry of the Environment, Climate Protection and the Energy Sector Baden-Wuerttemberg, Kernerplatz 9, 70182 Stuttgart, Germany.
} 
paper gives a brief survey of the system architecture and concentrates on the role of the KFUe with respect to the determination and evaluation of the radiological situation in the range of off-site emergency management. Due to its key role in that area, the dispersion calculation model included in the KFUe is described. A brief comparison to the recommendations of the ICRP (ICRP 2007; ICRP 2009) is given.

\section{System architecture}

The KFUe BW provides a measurement network and information system for operational parameters at the plant sites as well as radiological and meteorological measurements in their vicinity. All measurements in the vicinity like e.g. gamma dose rates, nuclide specific activity concentrations and meteorological parameters are performed independently from the operating company of the nuclear power plant. At the plant site, the originally measured data is extracted using isolating amplifiers and separate signal processors, which additionally enable a real-time access to the plant data. Complementary to the most important operational parameters like neutron fluxes, core temperatures, pressures, water levels, the emissions of radioactive particles to air and water are recorded. Altogether, about 5000 measurement time series are recorded with various time resolutions like e.g. one or ten minutes. Figure 1 provides an overview of the systems main components. Starting from the data acquisition from the own sensor network or data exchange with other measurement networks like the BfS, MADUK or the German weather service (DWD), which are managed by a communication server, the data is automatically validated and stored in a central database located at the State Institute for Environment, Measurements and Nature Protection (LUBW). In addition to the originally measured data, various algorithms are implemented. Examples are the

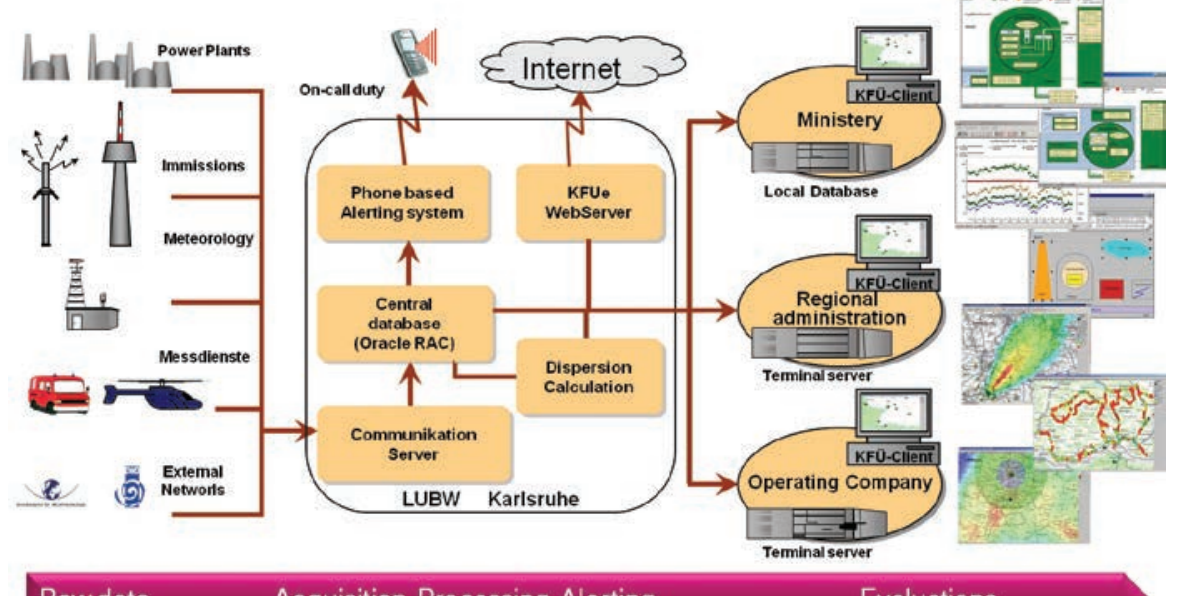

Raw data Acquisition, Processing, Alerting Evaluations

Figure 1 - Basic system architecture of the KFUe. 
nuclide inventory, turbulence parameters describing the planetary boundary layer, or dose rate net values. The main issue there is to prepare the necessary information for the dispersion calculation models which will be described below. In order to avoid incidents being unnoticed, an automatic alerting including on-call duty of the supervisory authority is implemented whenever threshold violations are detected by the system. Web servers provide information to the intranet and internet. Especially, a web system for the radiological situation report is attached. As indicated on the right side of Figure 1, the KFUe-system provides several methods to analyse and present the measured data as well as to ensure compliance of threshold limits and safety objectives. For this purpose, a special software - the client KFUe - has been developed. This tool allows for the necessary database queries and the presentation of the results in curves, tables or maps. In addition, it also serves for system administration tasks. In the last years, the KFUe increasingly exploits modern Internet technologies to optimize workflows and collaborations of all users and organizations involved. Therefore, a central KFUe portal provides the specific information for the relevant users at the different locations. Moreover, for training purposes, an application for mobile devices has been developed using cellular phone network.

In all parts of the central data centers, the data acquisition and networking of the nuclear reactor remote monitoring is designed for high availability. The computer clusters for the central data management and all key components in power plants and at the participating authorities are equipped with emergency power supplies to ensure continued operational availability. To avoid an overload or breakdown of the central servers in case of emergency, the architecture of KFUe provides a regular distribution of centrally stored data to the UM, enabling faster data access for the users. Concerning the ambient dose rate measurement network, a circuit-independent wireless sensor system with redundant receivers located close to the plant sites and on the Königstuhl and the Feldberg are used. Beyond Baden-Wuerttemberg, the KFUe system is deployed in Rhineland-Palatinate and the Kerntechnische Hilfsdienst GmbH (KHG), where the focus is on mobile measurements in emergency situations on behalf of the operating companies.

The KFUE also provides a realistic simulation environment to support regular exercises in which the interaction of the systems and organizations involved is trained (Wilbois et al., 2009). Whereas the plant scenario is usually based on the plant simulator, the environmental part is designed by using the dispersion calculation, which is used to fix the "measured" dose rates, depositions and concentrations including mobile measurements as a part of the exercise.

\section{Dispersion calculation (ABR)}

The name ABR is the abbreviation of the German word for dispersion calculation, "Ausbreitungsrechnung". The ABR is a modular system containing models to calculate the release, transport, and deposition of the radioactive particles, followed by dose calculations. The model includes complex three-dimensional 
meteorological data (measured data as well as forecasts) and the topography of the considered area.

The calculation of the wind regime is performed with a three-dimensional, so called diagnostic model, by interpolating the initial values, either from measurement devices or taken from a weather forecast, in horizontal and vertical direction for the underlying three-dimensional grid. In a second step the continuity equation is solved to ensure mass consistency. The stability of the atmosphere is described by a stability class parameter. Within the ABR, two different models are available to calculate the wind regime. The main difference between them is that one uses a Cartesian coordinate system and the second one uses a terrain following coordinate system.

The dispersion of particles in the atmosphere is described by the advectiondiffusion-equation. In the KFUe, a Monte-Carlo/Lagrange model approach, a statistical model where trajectories of the particles are calculated, is used. Whereas the advection part is determined by the wind regime described above, the turbulence part is parameterized by so-called dispersion parameters, either by Gifford (1976) or by Thomas et al. (1981). Both sets of parameters result from experiments and describe the standard deviation of the dispersion of the particle cloud, depending on the emission height and the distance from the emission point. In order to allow for different diffusion properties, the released nuclides are grouped into the four nuclide groups noble gas, aerosols, elementary and organic iodine. Currently, about 50 different nuclides are considered explicitly. The dry deposition of aerosols and iodine are mainly described by the deposition velocity factor and the terrain roughness factor with distinct formulas calculating the deposition velocity for small and large particles. Concerning the wet deposition of the particles, inhomogeneous distributed precipitation data, as determined at different locations including radar data, are taken into account. Chemical reactions between the radioactive particles and the rain are not considered in the current model for the washout coefficients. As a result of the calculation, three dimensional activity concentrations and two dimensional deposition values for the nuclides are available. The solution area of the ABR varies from $25 \mathrm{~km} \times 25 \mathrm{~km}$ up to $200 \mathrm{~km} \times 200 \mathrm{~km}$ using an equally spaced grid with mesh sizes from $100 \mathrm{~m}$ up to $1 \mathrm{~km}$. These fields are the starting point for the calculation of the doses.

The dose calculation consists of basically two models, the first one calculates the cloud radiation and the second one calculates the doses for different organs and age groups, based on the radiation of cloud (including inhalation) and the deposited particles on the ground. The calculation of the cloud gamma radiation is based on the model of adjoint fluxes (Sohn, 1994), describing the activity fraction of the gamma radiation from one point in the three-dimensional grid to one point on the ground, taking into account thirty gamma energy groups. Altogether, about 50 nuclides including the radiological most important ones are taken into account explicitly. Concerning the organ doses, the current dose rates as well as the time integrated doses are calculated, based on the activity concentration of the nuclides. The calculation of the doses for twenty-one different organs is based on dose rate coefficients taken from (BMU, 2005b). In order to simplify the comparison of 
ABR results with measured data and to possibly renormalize the source term of the calculations, in addition to the nuclide specific activity concentrations and depositions the local gamma dose rate is calculated too. The ABR provides integrated doses as well as dose rates for each time step which in combination with the use of prognostic source terms and weather forecasts provides the possibility to optimize possible strategies in case of a nuclear accident.

As already denoted above, various kinds of calculations are possible, which are managed and parameterized by a web user interface. The diagnostic calculation is based on the measured data at the plant site, mainly the emission rates, and the weather data in the surrounding area. Such a calculation starts automatically whenever a radiological alarm is raised by the system. If no measured data is available, the calculation can be parameterized manually where source term and meteorological data can be determined for each time step of the calculation. Alternatively, the emission data can be estimated by using prepared source terms, which refer to standard accident scenarios described in (BMU, 2004). Finally, based on the COSMO models of the DWD and prognostic source terms, prognostic calculations are available.

With respect to the degree of conformity to the ICRP recommendations the ABR behaves the same as that of the current national legislation in Germany. The methods for calculation of doses, especially the role of the effective dose are very close. To ensure conformity, basically the dose factors have to be adapted, if necessary. At present, the importance of considering avoided dose due to executed measures is emphasized. That kind of report is currently not included in the ABR even though it would be easy to provide. The application of these evaluations in the emergency organization of the UM is currently discussed. When the German law adopts the new ICRP recommendations concerning the residual dose, the system will be updated appropriately.

\section{The KFUe in off-site emergency management}

Authorities and operators are obliged to plan for nuclear emergencies. The KFUe provides various tools to assess the consequences of such an event already in the prerelease-phase in order to take appropriate measures to soften the consequences for the inhabitants. For this purpose, in addition to the UM, the LUBW and operating companies, the chief district authority agencies in Freiburg, Karlsruhe and Stuttgart being responsible for emergency protection are connected to the KFUe. Direct access to the electronic situation report system of the authority is possible. The implementation of roles and measures follows the recommendations of the national guidelines in Germany. The first important task of preventive civil protection is to determine the potentially affected areas caused by the release of radioactive materials using the prognostic dispersion calculation. In the release phase, the radiological situation is merely determined by stationary and quasistationary immission measurements which track the actual course of a possible release and allow for immediate adjustments of possible measures. After the release, mobile 
measuring teams of LUBW, the operating company, the Federal Office for Radiation Protection (BfS) and the Fire Department are gathering detailed radiological data in and around the affected area in order to create the data base for appropriate decisions to protect the population. This data is processed in the KFUe and analyzed together with the stationary online-measurements providing a complete overview of the radiological situation (see Fig. 2).

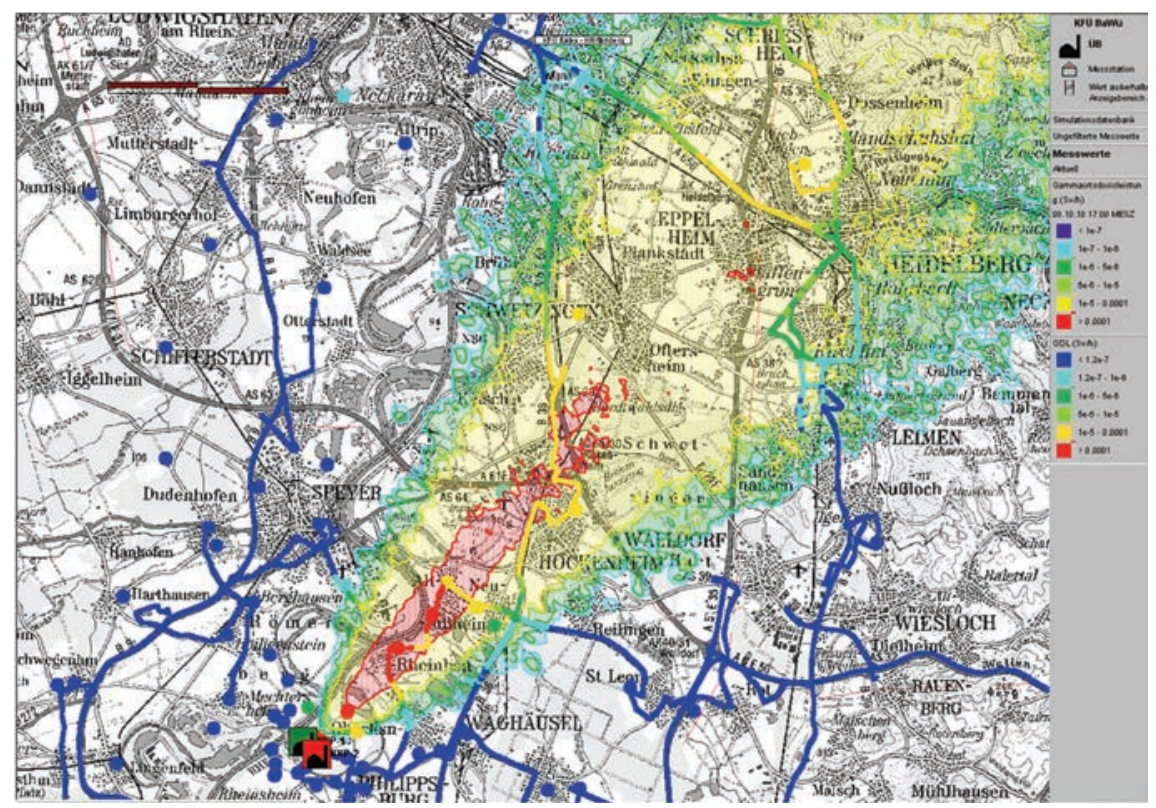

Figure 2 - Example of a combined evaluation of the KFUe. Based on a severe accident scenario for the nuclear power plant in Philippsburg the results of simulated stationary and mobile measurements are displayed in combination with the dispersion calculation (Scheuermann et al., 2011)

Based on the current radiological situation, the most important part of the UM is to recommend the appropriate measures. The focus is on the protection of people in emergency exposure situation caused by an accident in a nuclear power plant, so recommendations of ICRP Publication 109 apply here. The application of the ICRP approach concerning the residual doses is currently discussed within the German Ministry for environment and will be applied in the system immediately after the corresponding law is changed. Concerning off-site emergency management at present, there are three different measures which would be recommended by the UM. Table I lists the measures and the reference level of the corresponding doses.

The main problem is, however, to deliberate whether the measure is available and meaningful in the context of the current situation. As an example, it may be counterproductive to evacuate in case of a forthcoming release. The determination of the personal doses is part of the concept of the emergency stations, where at present no detailed information concerning the radiological situation is available. 
Combining the measured data and the results of diagnostic dispersion calculations as provided by a system like the KFUe could help to optimize this process.

Table I

Measures and reference levels used by the UM.

\begin{tabular}{ccc} 
Measure & Dose & Reference level \\
Intake of iodine tablets & $\begin{array}{c}\text { thyroid inhalation dose } \\
\text { (7 days integration time includ- } \\
\text { ing dose commitment) }\end{array}$ & $\begin{array}{c}50 \mathrm{mSv} \text { (children, pregnant } \\
\text { women) }\end{array}$ \\
Sheltering & $\begin{array}{c}\text { Effective dose (children) } \\
\text { (7 days integration time includ- } \\
\text { ing dose commitment) }\end{array}$ & $10 \mathrm{mSv}$ \\
Evacuation & $\begin{array}{c}\text { Effective dose (children) } \\
\text { (7 days integration time includ- } \\
\text { ing dose commitment) }\end{array}$ & $100 \mathrm{mSv}$ \\
\hline
\end{tabular}

\section{REFERENCES}

BMU (Bundesministerium für Umwelt, Naturschutz und Reaktorsicherheit) (2004) Leitfaden für den Fachberater Strahlenschutz der Katastrophenschutzleitung bei kerntechnischen Notfällen, Bonn.

BMU (Bundesministerium für Umwelt, Naturschutz und Reaktorsicherheit) (2005a) "Neufassung der Rahmenempfehlungen für die Fernüberwachung von Kernkraftwerken" - RdSchr. d. BMU v. 12. 8. 2005 - RS II 5 - 17031, Bonn.

BMU (Bundesministerium für Umwelt, Naturschutz und Reaktorsicherheit) (2005b) Entwurf der Allgemeinen Verwaltungsvorschrift zu $§ 47$ StrlSchV, Bonn.

Gifford F.A. jr. (1976) Turbulent diffusion-typing schemes - a review, Nucl. Safety 17, 68 .

ICRP Publication 103 (2007) The 2007 Recommendations of the International Commission on Radiological Protection, Ann. ICRP 37(2-4).

ICRP Publication 109 (2009) Application of the Commission's Recommendations for the Protection of People in Emergency Exposure Situations, Ann. ICRP 39 (1).

Scheuermann W. et al. (2011) ABR - Einsatz des Systems “Ausbreitungsrechnung” bei radiologischen Katastrophenschutzübungen. In: Mayer-Föll, R, Ebel, R., Geiger, W. (Eds.): F+E-Vorhaben KEWA - Kooperative Entwicklung wirtschaftlicher Anwendungen für Umwalt und Verkehr in neuen Verwaltungsstruckturen, Phase VI 2010/2011, KIT Scientific Publishing.

Sohn A. et al. (1994) Ein neuer Dosismodul zur Berechnung und Darstellung der effektiven Dosis und von 21 Organdosen für die Dosispfade Submersion, Inhalation und Bodenstrahlung, Universität Stuttgart, IKE 6 UM 3.

Thomas P. et al. (1981) Experimental determination of the atmospheric dispersion parameters at the Karlsruhe nuclear research center for $60 \mathrm{~m}$ and $100 \mathrm{~m}$ 
emission heights, part 1 measured data, Kernforschungszentrum Karlsruhe, KfK 3090.

Wilbois T. et al. (2009) SIM-NOT - Einsatz von Simulationen im radiologischen Notfallschutz, In: Mayer-Föll, R., Keitel, A., Geiger, W.; Hrsg.: F+EVorhaben KEWA - Kooperative Entwicklung wirtschaftlicher Anwendungen für Umwelt und Verkehr in neuen Verwaltungsstrukturen, Phase IV 2008/09, Forschungszentrum Karlsruhe, Wissenschaftliche Berichte, FZKA 7500, 71-80. 